\title{
A simple microfluidic aggregation analyzer for the specific, sensitive and multiplexed quantification of proteins in a serum environment
}

\author{
Nitin K. Rajan ${ }^{\dagger}$, Sukumar Rajauria ${ }^{\ddagger}$, Tyler Ray॥, Sumita Pennathur" and Andrew N. Cleland ${ }^{\#, *}$ \\ $\dagger$ Department of Physics, University of California Santa Barbara, Santa Barbara CA 93106 \\ † HGST Western Digital, San Jose Research Center, San Jose, CA 95138 \\ || Department of Mechanical Engineering, University of California Santa Barbara, Santa Barbara CA \\ 93106 \\ \# Institute for Molecular Engineering, University of Chicago, Chicago IL 60637
}

\begin{abstract}
Portable and low-cost platforms for protein biomarker detection are highly sought after for point of care applications. We demonstrate a simple microfluidic device for the rapid, electrically-based detection of proteins in serum. Our aggregation analyzer relies on detecting the protein-induced aggregation of submicron particles, using a one-step procedure followed by a fast, particle-by-particle measurement with a very high count rate. This enables the rapid and precise quantification of $C$-Reactive protein levels, within the clinically relevant range, using unprocessed human serum and a disposable microfluidic device; no optics are involved in the implementation. Due to the single particle detection format and the use of microfluidics, only a small volume of serum $(\sim 50 \mathrm{~nL})$ is needed to complete the analysis. The method can be easily extended to multiplexed biomarker detection by combining an assay using differently sized particles, each targeting a separate protein. We illustrate this by using two sizes of latex beads and demonstrating the simultaneous detection of two different proteins in a serum environment with minimal cross-interference. This confirms that our aggregation analyzer platform provides a simple and straightforward method for multiplexed biomarker detection in a low cost, portable design.
\end{abstract}

Keywords: resistive pulse sensing, microfluidics, lab-on-chip, particle immunoassay, label-free biosensing

* Corresponding-Author (E-mail: anc@uchicago.edu) 


\section{INTRODUCTION}

Rapid, low-cost immunoassays with multiplexing capabilities, able to detect multiple protein targets directly in complex bodily fluids such as serum, are highly sought after for point-of-care applications, especially in the context of early patient screening (Sista et al., 2008; Wang, 2006) and accurate diagnostics in resource limited settings (Hay Burgess et al., 2006; Yager et al., 2008). Diagnostics based on immunological tools such as antibody-antigen based capture or sandwich assays (e.g. enzyme linked immunosorbent assay, ELISA) have become the gold standard, because of their high specificity and sensitivity (Wilson, 2013). However, such assays are not straightforward, requiring lengthy incubation times, specialized technicians and numerous wash steps and reagents to generate an optical signal (Ozkumur et al., 2008). Point-of-care devices require a simple measurement scheme that can yield accurate and sensitive results rapidly (Tudos et al., 2001). Here, we demonstrate a microfluidic aggregation analyzer for the quantitative detection of proteins, combining the low cost and ease of use of latex immunoagglutination (LIA) tests (Molina-Bolivar and Galisteo-Gonzalez, 2005; Wilson et al., 1960) with high sensitivity in an integrated on-chip, electrical detection platform.

The microfluidic aggregation analyzer is based on resistive pulse sensing (RPS) (Coulter, 1956; Deblois and Wesley, 1977), which provides a simple electrical detection and size measurement method, where single particles flowing through a narrow constriction change the electrical resistance of the constriction by an amount proportional to the volume of the particle. With advances in microfluidic technology, a number of on-chip RPS architectures have been proposed for the counting and sizing of submicron and micron-sized particles (Carbonaro and Sohn, 2005; Rodriguez-Trujillo et al., 2007; Sridhar et al., 2008; Zhe et al., 2007). Previously, we demonstrated a particle analyzer with high throughput and very accurate nanoscale size characterization (Fraikin et al., 2011) and in this work, the same high throughput and sizing accuracy is leveraged for the rapid and precise quantification of LIA utilizing sub-micron particles, such as those commonly used in commercial agglutination kits intended for turbidity measurements (Fujita et al., 1994; Phillip and Andrews, 1987). High throughput is a critical aspect of using RPS for 
aggregation analysis because it enables the analysis of a large number of particles in a short time, which therefore allows more accurate quantification of dimers, trimers and so on, as a result of counting statistics. This makes it possible to rapidly and accurately quantify aggregation assays in a way that is not possible with other RPS architectures that are bandwidth and therefore throughput limited.

The microfluidic device is simple to fabricate, as it is very inexpensively replicated from a re-useable SU8 (MicroChem Corp.) patterned mold, with features defined by standard optical lithography. Using this device, we carry out the detection and quantification of C-reactive protein, a biomarker for inflammation and cardiovascular disease, using functionalized beads from a commercial immunoturbidimetric assay (hsCRP, Diazyme Inc.). Results show an improved detection limit with our platform as compared to optically-based detection, with a much smaller volume of both sample $(50 \mathrm{~nL})$ and reagents used compared to the optical method. We also demonstrate the potential for the aggregation analyzer to perform multiplexed detection and quantification of proteins in a serum environment, by employing two different bead sizes, functionalized with different protein receptors. The single particle detection format allows the signal to be gated and the different proteins analyzed separately, in a single measurement run. Our results furthermore show minimal cross-interference, highlighting the flexibility of this approach, and underlining the potential for this platform to provide simple, low-cost and accurate point-of-care detection of multiple protein biomarkers.

\section{MATERIALS AND METHODS}

\subsection{Device Fabrication}

The microfluidic devices were fabricated using cast polydimethylsiloxane (PDMS) from a $100 \mathrm{~mm}$ silicon master mold, the mold defined by photolithographically-patterned SU-8 (Microchem Corp.) features. The mold was fabricated using SU-8 of two different thicknesses. Alignment marks were first patterned using a gold lift-off procedure. The micro-constriction (MC) and fluidic resistor (FR) were patterned next in a $2.5 \mu \mathrm{m}$ thick layer of SU-8. The MC was designed to be $2.5 \mu \mathrm{m}$ long and wide 
whereas the FR consisted of 17 parallel ribs, each $50 \mu \mathrm{m}$ long and $3 \mu \mathrm{m}$ wide. The remainder of the channel was patterned in a second layer of SU-8 which was $8 \mu \mathrm{m}$ thick, and included $3 \mu \mathrm{m}$ long and wide filtering posts, with a $3 \mu \mathrm{m}$ gap between neighboring posts. To make the PDMS device, two-part RTV615 (Momentive Inc.) was mixed in a ratio of 10 parts A to 1 part B, then degassed for $1 \mathrm{hr}$. The mixture was poured over the silicon/SU-8 mold and cured for $45 \mathrm{~min}$ on a hot plate at $110^{\circ} \mathrm{C}$. The cured PDMS was then carefully peeled off, each device cut into $2.5 \mathrm{~cm}$ by $2 \mathrm{~cm}$ rectangles, and holes roughly $1 \mathrm{~mm}$ in diameter were punched for the inlet and outlet ports. Each PDMS device was then sonicated in isopropanol for 3 minutes and gently dried with nitrogen. The second component of the AAC is the glass substrate containing the sensing electrode, which was fabricated by a standard gold lift-off process on a $100 \mathrm{~mm}$ borosilicate glass wafer, which was subsequently diced into rectangular pieces containing a single sensing electrode each. The PDMS was irreversibly bonded to the glass piece by exposing both surfaces to UV-generated ozone (UVP PR-100) for 15 minutes, followed by bonding and heating the final PDMS-glass chip on a hot plate at $110^{\circ} \mathrm{C}$ for $5 \mathrm{~min}$. The PDMS-glass chip was then affixed and wirebonded to a printed circuit board (PCB) that included a voltage follower (LT1012 op-amp, Linear Technology). The voltage signal was then amplified by a low noise voltage amplifier (Stanford Research Systems, SR560) and the 10x amplified signal digitized by a Tektronix oscilloscope (DSO6032A), controlled and read-out using a computer running a custom Matlab script.

\subsection{Materials Used}

PDMS was obtained from Momentive Inc as a two part kit (RTV615A and B). $100 \mathrm{~mm}$ silicon wafers and glass (borosilicate) wafers were obtained from University Wafer. 10X Phosphate Buffered Saline (PBS) was purchased from Fisher Scientific Inc. and subsequently diluted 10-fold before use with the AAC and the bead assays. The polystyrene beads for the size calibration were obtained from Polysciences Inc. whereas the streptavidin beads were obtained from Spherotech Inc. (SVFP-0552-5). The biotin beads were purchased from Life Technologies (Fluospheres F-8768). The commercial turbidimetric assay (DZ135A) was purchased from Diazyme Inc. and consisted of the anti-CRP coupled beads as well as 
different levels of CRP spiked serum for control testing. Biotinylated BSA was purchased in lyophilized form from Sigma Aldrich and finally, streptavidin coated nanorods were obtained from Nanopartz Inc. (C12-25-700-TS-50)

\subsection{Streptavidin Bead Assay}

$10 \mu \mathrm{L}$ of streptavidin beads (Spherotech Inc., $0.45 \mu \mathrm{m}$ diameter) at an original concentration of $1.5 \times 10^{10}$ $/ \mathrm{mL}$ was added to $500 \mu \mathrm{L}$ of unit concentration PBS containing $0.1 \%$ Tween-20 (PBST) and thoroughly mixed by vortexing for $30 \mathrm{~s}$. Then, $10 \mu \mathrm{L}$ of various concentrations of the biotin-BSA solution in unit concentration PBST was added. The mixture was incubated at room temperature for $20 \mathrm{~min}$ before flowing through the aggregation analyzer chip (AAC), using an inlet port pressure of $2.0 \mathrm{psig}$ and an outlet port pressure of 0 psig.

\subsection{Diazyme hsCRP Assay}

Following the manufacturer's protocol for immunoturbidimetric measurements, $50 \mu \mathrm{L}$ of anti-CRP beads $\left(\sim 1.5 \times 10^{10} / \mathrm{mL}\right.$, approx. $370 \mathrm{~nm}$ diameter) were added to $300 \mu \mathrm{L}$ of PBST before adding $5 \mu \mathrm{L}$ of the serum sample containing CRP and incubating for $20 \mathrm{~min}$ at room temperature. The mixture was then flowed through and analyzed in the AAC to determine the aggregation ratio. We note the AAC only requires a very small fraction of the total prepared volume of reagent-serum mixture.

\subsection{Multiplexed Bead Assay}

$20 \mu \mathrm{L}$ of the anti-CRP beads were combined with $10 \mu \mathrm{L}$ of the biotin beads (Life Technologies, Fluospheres F-8768 -- 3×10 $10 / \mathrm{mL}, 1.0 \mu \mathrm{m}$ diameter) and $300 \mu \mathrm{L}$ of PBST. The mixture was mixed by vortexing for $30 \mathrm{~s}$. For the assay calibration $5 \mu \mathrm{L}$ of sample were added, which either contained no nanorods or the equivalent concentration of $290 \mathrm{nM}$ of streptavidin. The bead-sample mixture was incubated at room temperature for 20 min followed by measurement using the AAC. 


\subsection{Experimental Setup}

The principle of the aggregation assay is shown schematically in Figure 1a. The typical response curve (percentage of aggregates as a function of antigen concentration) is given by the Heidelberger-Kendall (H-K) curve (Wiklund et al., 2005), which resembles a skewed Bell curve with three distinct regimes, namely antigen deficit, equivalence and antigen excess. In the antigen deficit regime (Figure 1a, Panel 1), few analyte molecules are present, so most beads with antibodies are not bound to antigen, leaving most of the beads as monomers with a low percentage of small aggregates (dimers and some trimers). As the analyte concentration is increased, both the number and size of the aggregates increase, until the equivalence regime (Panel 2) is reached, where the antigen molecules bind to half the available number of antibody binding sites. This condition results in the highest aggregation percentage and a peak in the $\mathrm{H}-\mathrm{K}$ curve. As the analyte concentration is increased further, the bead binding sites become saturated (antigen excess regime, Panel 3) and the degree of aggregation decreases with increasing analyte. Thus, one major limitation of aggregation assays is that two different concentrations of analyte (antigen) can produce the same aggregation signal. The dynamic range of such an assay is therefore limited at the high concentration end by the equivalence point, when half the available binding sites are bound to antigen (Singer et al., 1973).
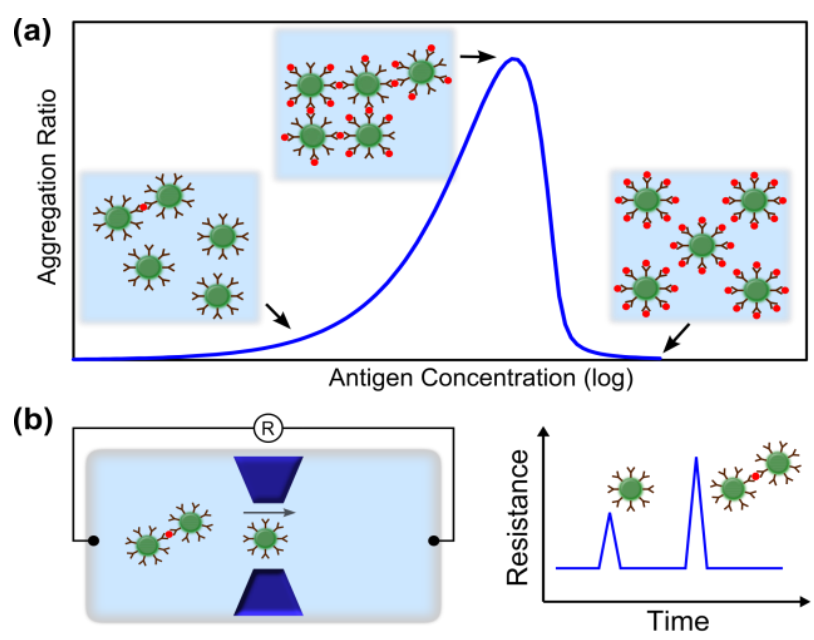

Figure 1 
The measurement scheme of our aggregation analyzer chip (AAC) is indicated in Figure 1b, in which individual particles are detected and their size measured using resistive pulse sensing (RPS) (Coulter, 1956; Deblois and Wesley, 1977). The microfluidic circuit in the AAC is filled with an aqueous conducting solution such as a dilute solution of $\mathrm{NaCl}$ in water, saline, or saline-containing buffer. This solution is made to flow through a narrow micro-constriction in the circuit; particles entrained in the flow pass through the constriction, displacing the fluid in the constriction, and thus increasing the device's electrical resistance. This change in resistance is proportional to the volume of the particle; we note this occurs regardless of whether the particle itself is metal or dielectric, as the Debye layer surrounding the particle prevents electrical conduction through the particle itself. A monomer bead can thus be distinguished from a bead dimer by the magnitude of the resistive change, which is detected as a change in the voltage between the micro-constriction and a biasing fluidic resistor; this is shown schematically in Figure 1b. The population of monomers, dimers, trimers, and so on, in both the absence and presence of the analyte, can thereby be measured and compared, which allows a quantitative measure of the degree of analyte-induced aggregation. We note that the ability to precisely distinguish particle sizes is crucial for sensitively distinguishing monomers from their aggregates. Furthermøre, the high measurement bandwidth afforded by the electrical design allows for the rapid counting of low concentrations of aggregates, thus enabling the degree of aggregation to be ascertained with small error (large counting statisties) in a short measurement time. Furthermore, the excellent time resolution (large measurement bandwidth) of our device enables the high-speed counting of particles, yielding small statistical errors due to the resulting large count numbers that can be achieved in a relatively short measurement time.

A schematic of the AAC device is shown in Figure 2a, which comprises microfluidic channels filled with e.g. unit concentration phosphate buffered saline containing $0.1 \%(\mathrm{v} / \mathrm{v})$ Tween-20 (PBST). A voltage bias $\left(V_{b}=15 \mathrm{~V}\right)$ is applied between two silver-silver chloride electrodes placed in the solution at the inlet and outlet regions. A patterned gold electrode is used as a sensing electrode, capacitively-coupled through the Debye layer to the conducting solution. On the left of the sensing region is a fluidic resistor (optical 
micrograph shown in Figure 2a), comprising 17 parallel channels $(50 \mu \mathrm{m}$ long, $3 \mu \mathrm{m}$ wide and $2.5 \mu \mathrm{m}$ high). On the right of the sense electrode is the micro-constriction with all dimensions being $2.5 \mu \mathrm{m}$ (optical micrograph in Figure 2a). The cross-hatched blocks indicate filter regions, formed by rectangular posts $8 \mu \mathrm{m}$ high with $3 \mu \mathrm{m}$ separation in both lateral dimensions.

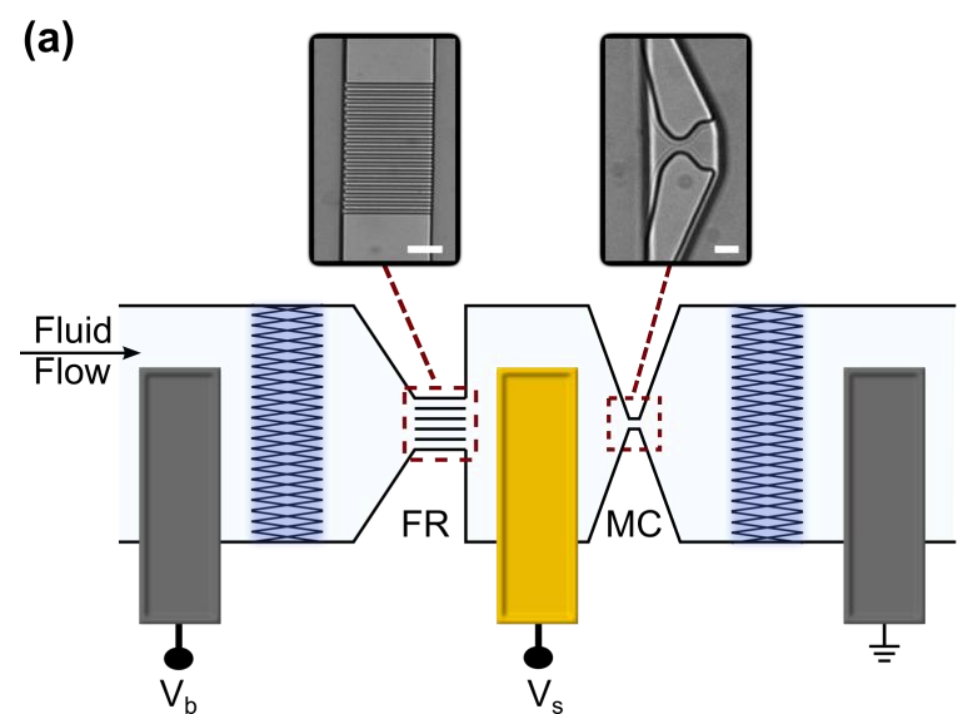

(b)

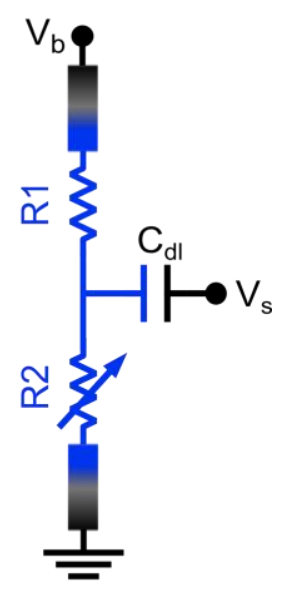

Figure 2

The working principle of this device architecture is best understood using the equivalent potential divider circuit shown in Figure 2b.The electrical resistance of the solution in the fluidic circuit to the left of the sensing electrode (including the fluidic resistor) is modeled as a single resistance R1 and the resistance of the buffer solution in the micro-constriction and the channel to the right of the sensing electrode is modeled as a variable resistor R2. A particle passing through the micro-constriction increases the resistance R2, which alters the potential of the solution in contact with the sense electrode, generating a voltage pulse at the sense electrode. This voltage signal is passed to a proximal unity-gain voltage follower amplifier, band-pass filtered $(1 \mathrm{kHz}-1 \mathrm{MHz})$, amplified (10x) by a low-noise voltage amplifier, and finally recorded by an oscilloscope as the output voltage $V_{\text {out }}$. The advantage of using the fluidic voltage divider configuration is that it easier to achieve large bandwidth measurements (Fraikin et al., 2011). 

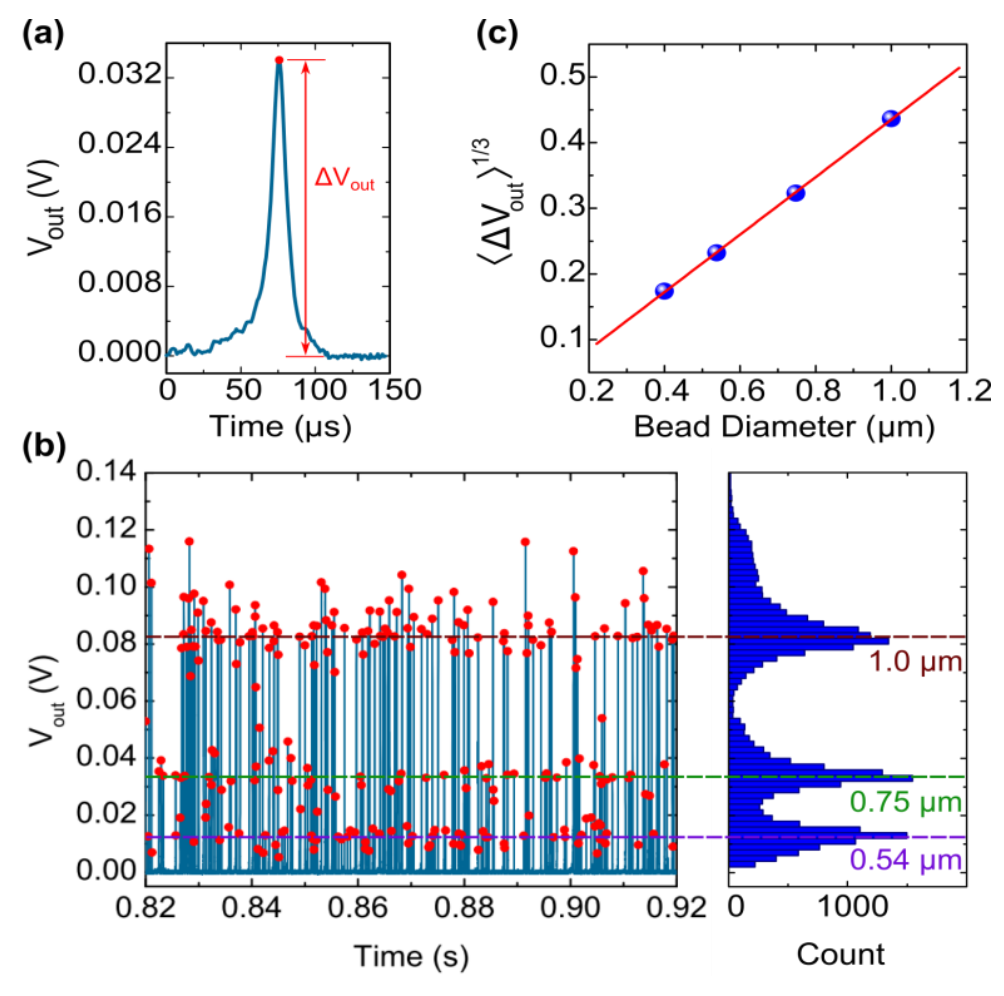

Figure 3

An individual voltage pulse is shown in Figure 3a. This pulse is due to the passage of a $0.75 \mu \mathrm{m}$ bead through the AAC's micro-constriction, highlighting both the excellent signal-to-noise ratio as well as the time resolution of the device. From the voltage pulse, the peak signal $\Delta V_{\text {out }}$ is extracted as shown in Figure 3a. Figure $3 \mathrm{~b}$ shows a representative time trace generated by a mixture of three polystyrene bead sizes (diameters of $0.54,0.75$ and $1.0 \mu \mathrm{m}$ ) passing through the micro-constriction. The peaks in the time trace were identified (red circles) and a histogram of the measured peak voltage values was generated. The three signal levels can be clearly distinguished and the histogram of the combined data accumulated for a few seconds displays the three peaks corresponding to the different bead populations, also shown in Figure $3 \mathrm{~b}$. The histogram highlights the ability of the AAC to detect and distinguish different particle 
sizes, providing the basis for using this instrument as both a sensitive and quantitative aggregation detector.

A tri-modal Gaussian fit of the histogram data in Figure $3 \mathrm{~b}$ allows us to extract the mean values $\left\langle\Delta V_{\text {out }}\right\rangle$ corresponding to each of the three bead sizes. These mean values are proportional to the ratio of the particle volume to the micro-constriction volume (Deblois and Bean, 1970), as seen from the linear relationship between $\left\langle\Delta V_{\text {out }}\right\rangle^{1 / 3}$ and the bead diameter displayed in Figure 3c.

\section{RESULTS AND DISCUSSION}

\subsection{Proof-of-principle using biotin-streptavidin model system}

In order to demonstrate that our platform is indeed able to accurately characterize the aggregation process, we measured the aggregation of streptavidin (SAv) functionalized beads due to the addition of biotinylated bovine serum albumin (BBSA) in unit concentration phosphate buffered saline with $0.1 \%$ (v/v) Tween-20 (PBST). We compare the results from the AAC to results obtained from absorbance measurements carried out using a UV-Vis spectrophotometer. Figure 4a displays the signal distribution for $0.45 \mu \mathrm{m}$ diameter SAv beads without any BBSA added (negative control), showing a clear monomer peak as well as a small dimer peak from the auto-aggregation of SAv beads, with negligible higher-order aggregates. Upon the addition of BBSA $(1 \mu \mathrm{g} / \mathrm{mL})$, the distribution changes significantly as can be seen in Figure 4b. The majority of the beads are still in monomer form but now significant dimer and trimer peaks are clearly visible as well as higher order aggregates. The number of monomer events recorded within the same time frame decreases, indicating that the concentration of monomers is reduced as the individual SAv beads combine to form aggregates. In order to quantify the aggregation, we define a threshold signal level $\left(V_{t h}=0.011 \mathrm{~V}\right)$ and calculate the aggregation ratio as the number of events above $V_{t h}$ divided by the total number of recorded events. This procedure was repeated for different concentrations of BBSA; the results are plotted in Figure 4d (red points). The shape of the response curve 
follows the expected Heidelberger-Kendall curve (Heidelberger and Kendall, 1935) and highlights the three regimes illustrated in Figure 1.
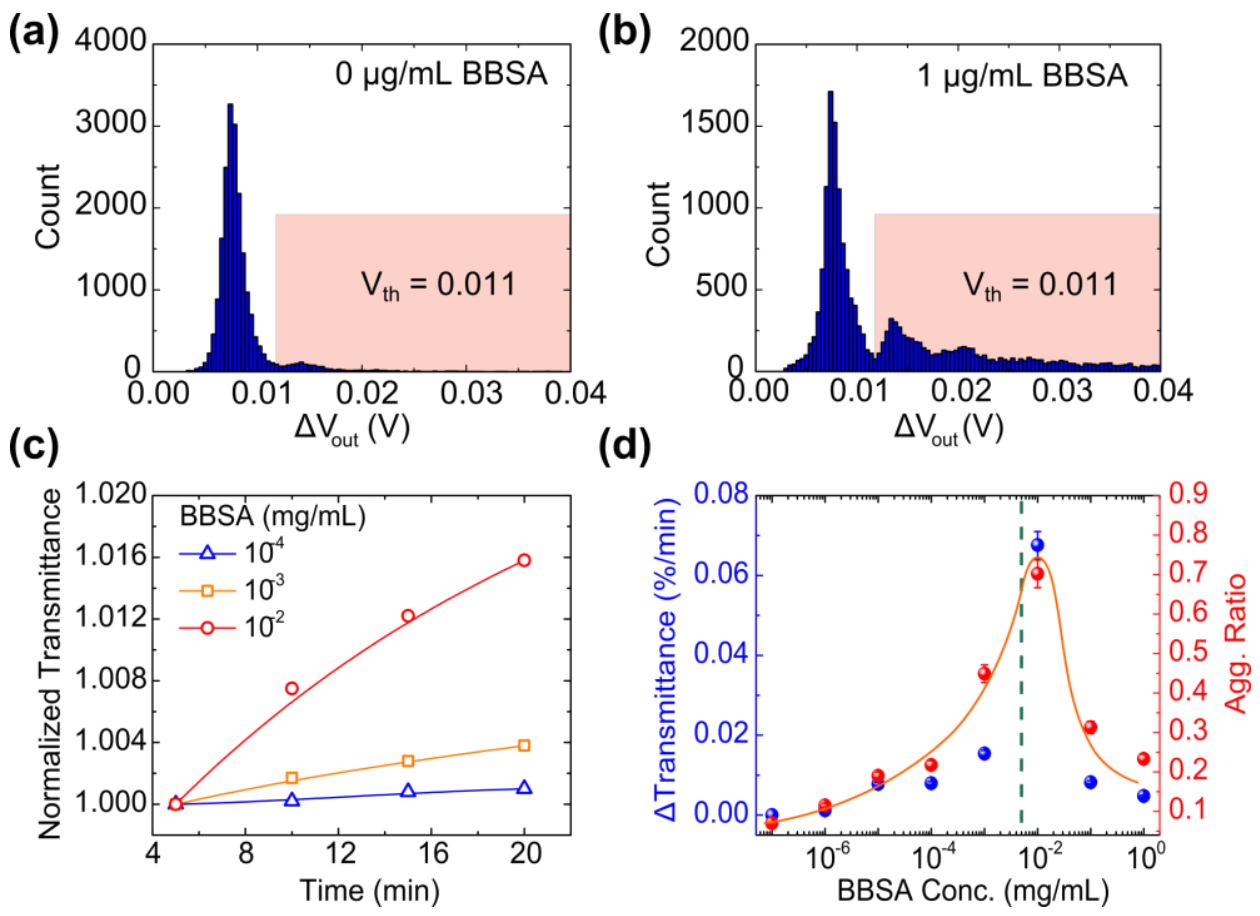

Figure 4

In order to confirm the accuracy of our results, we used a UV-Vis spectrophotometer (Shimadzu

UV3600) to perform turbidimetric analysis by measuring changes in transmittance at $570 \mathrm{~nm}$ due to the addition of BBSA to the same SAv bead population (changes in transmittance are reported rather than absolute transmittance, as changes are more reproducible than the absolute values). Figure $4 \mathrm{c}$ shows the change in transmittance with time for three concentrations of BBSA. As the concentration of BBSA is increased, there are more analyte molecules per bead and the aggregation process proceeds faster (within the antigen deficit regime), resulting in a faster increase in transmittance. For turbidimetric assays, the change in transmittance (or absorbance) in a time interval of 1 minute is determined and plotted for each analyte concentration. The rate of change of transmittance is measured from the change of transmittance between $\mathrm{t}=5 \mathrm{~min}$. and $\mathrm{t}=10 \mathrm{~min}$. The results are plotted in Figure $4 \mathrm{~d}$ (blue points). These are seen to be in 
excellent agreement with the AAC results, demonstrating that our platform provides an accurate way to quantitatively measure and characterize the aggregation process. The peak of the response curve in Figure $4 \mathrm{~b}$ indicates the equivalence point, where the fractional occupation of antibody sites by BBSA is $50 \%$. This peak value (equivalence concentration), at about $0.01 \mathrm{mg} / \mathrm{mL} \mathrm{BBSA}$, is consistent with the estimated value of $0.005 \pm 0.002 \mathrm{mg} / \mathrm{mL}$, calculated from the biotin-FITC binding capacity of the SAv beads (Spherotech Inc. datasheet) and an estimate of 12 moles of biotin per mole of BBSA (Sigma Aldrich).

\subsection{Characterization of C Reactive Protein assay}

To show the applicability of our device to existing immunoturbidimetric assays, we carried out aggregation tests using a commercial assay for the high sensitivity detection of C-reactive protein (hsCRP assay, Diazyme Inc.). C-reactive protein (CRP) is produced by the liver; in the event of an inflammation or infection, the level of CRP in the blood rises. There have been studies that show a correlation between elevated serum CRP levels and a higher risk of cardiovascular disease (Pearson et al., 2003; Pepys and Hirschfield, 2003). It is therefore important to be able to accurately measure low levels of CRP for patient screening as well as for treatment monitoring. The aggregation assay consists of latex beads that have been coated with antibodies specific to CRP (anti-CRP); the analyte comprises human serum samples with different CRP concentrations . 
(a)

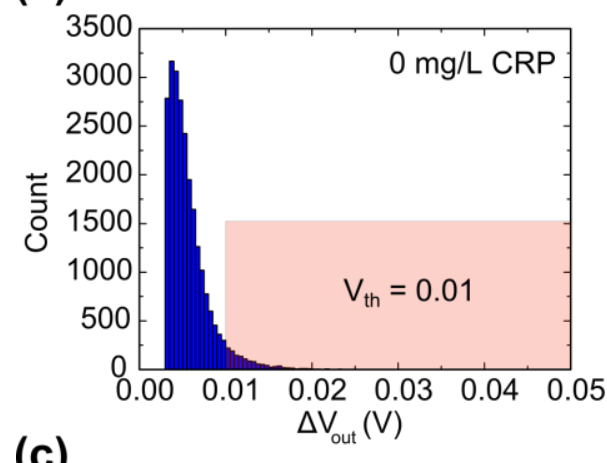

(c)

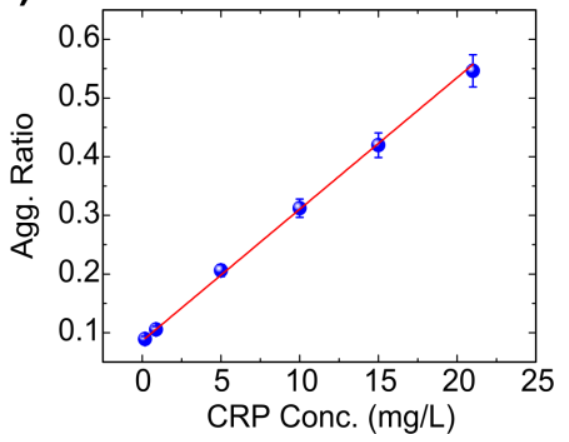

(b)

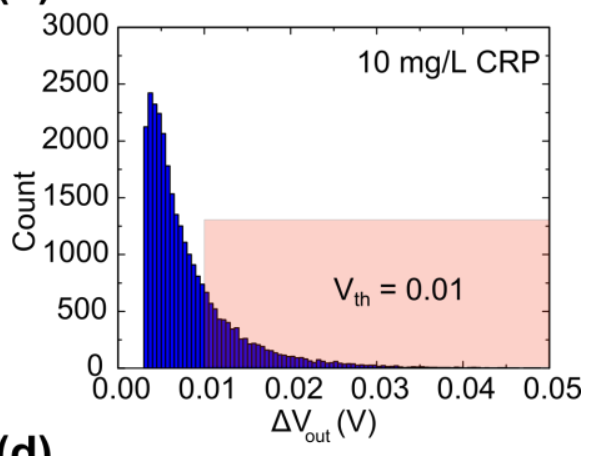

(d)

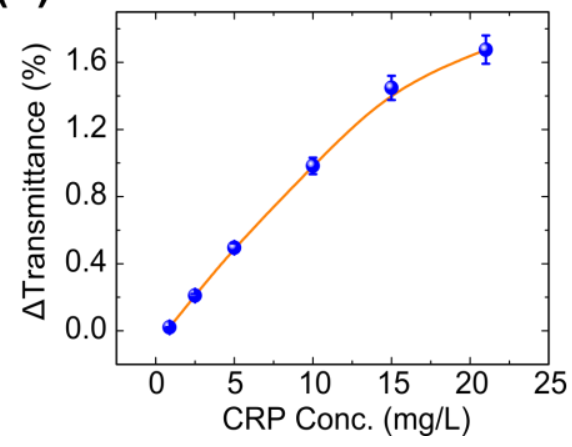

Figure 5

Figure 5a shows the histogram generated by the AAC for the size distribution of the anti-CRP beads used in the assay, in the absence of CRP serum, with an incubation time of $20 \mathrm{~min}$. The primary peak corresponds to a mean bead diameter of approximately $370 \mathrm{~nm}$; we note the distribution of particles is quite broad, with a smooth tail extending to larger bead diameters. We select an aggregation threshold of $V_{t h}=0.01 \mathrm{~V}$, yielding a baseline aggregation ratio (ratio of the number of events above threshold to the total number of events, within the user-defined analysis window) of approximately $5 \%$. Figure $5 \mathrm{~b}$ shows the signal distribution after the addition of a serum sample containing a CRP concentration of $10 \mathrm{mg} / \mathrm{L}$, followed by an incubation time of $20 \mathrm{~min}$. Contrary to the results obtained using the SAv beads, dimer and trimer peaks are not clearly distinguishable. Instead, the histogram distribution broadens and becomes more skewed to the right, indicating the presence of higher order aggregates; the dimer and trimer peaks are not distinguishable due to the broad polydispersity of the original anti-CRP bead population. We use the same protocol as before to calculate the aggregation ratio, based on the threshold voltage of $0.01 \mathrm{~V}$, and plot the aggregation ratio as a function of CRP concentration in Figure 5c. UV transmittance 
measurements for the hsCRP kit are shown in Figure 5d, representing the change in transmittance calculated from measurements made $5 \mathrm{~min}$. and $10 \mathrm{~min}$. after addition of CRP. We determine the limit of detection (LOD), the lowest CRP concentration which resulted in a positive change in transmittance, 2 standard deviations above the blank measurement mean value, to be $0.88 \mathrm{mg} / \mathrm{L}$; the highest concentration investigated was $21 \mathrm{mg} / \mathrm{L}$. The clinical range for CRP falls between 1 and $10 \mathrm{mg} / \mathrm{L}$. The kit is rated for a LOD of $0.2 \mathrm{mg} / \mathrm{L}$; this is specified for a measurement carried out at $37^{\circ} \mathrm{C}$, which likely enhances the aggregation rate. The same bead assay measured using the AAC yields a linear calibration curve with a lower LOD of $0.18 \mathrm{mg} / \mathrm{L}$ for measurements carried out at room temperature. The aggregation signal can potentially be increased by incubating for a longer time, as this is an end-point measurement as opposed to the rate measurement used for turbidimetry. It is clear that the AAC is more sensitive and more importantly requires no bulky optical setups since it relies on electrical signals only. Another significant advantage of the AAC is that repeated user calibration is not necessary. For the same microfluidic chip, with the same experimental conditions and batch of functionalized beads, a single calibration curve is sufficient because the measured signal only depends on the physical dimensions of the molded microconstriction. This calibration curve could be supplied as a specification of the assay and the user's measurement data for a particular bead set would lie on the curve, as one can see from the low standard deviations and repeatability of our measurements, carried out using separate chips. Perhaps most significantly, the microfluidic AAC requires only $50 \mathrm{~nL}$ of serum sample for one measurement and a total volume of sample plus reagents (beads and diluent) of $2 \mu \mathrm{L}$. By comparison, the turbidimetric measurement requires a much larger total volume of $250 \mu \mathrm{L}$ and utilizes $5 \mu \mathrm{L}$ of serum sample, since this is a bulk measurement technique. This is of considerable utility when analyte samples are precious.

\subsection{Multiplexed Analyte Detection}

Another advantage of the AAC platform, as compared to turbidimetric analysis, is the ability to perform multiplexed analyte sensing. This can be implemented by employing a mixture of different bead sizes, with each bead size functionalized for a particular target. Since our aggregation analysis counts and sizes 
the individual particles and aggregates, different bead populations can be distinguished, gated and analyzed for the separate aggregation ratios produced by a single sample. Being able to simultaneously detect and measure multiple serum proteins could allow for more accurate and specific clinical diagnoses. In the case of acute myocardial infarction, for instance, CRP detection could be performed in parallel with other cardiac markers, such as cardiac troponin and myoglobin, markers that are typically measured together to provide for a more complete and accurate diagnosis (Sabatine et al., 2002; Wolf et al., 2004). A single multiplexed assay of this type could then be performed in a single measurement, using a very small quantity of patient serum.

To demonstrate the feasibility of multiplexed detection, we combined the anti-CRP beads (approximately $370 \mathrm{~nm}$ in diameter) with a population of biotin-coated beads (1.0 $\mu \mathrm{m}$ dia.). With such different sizes, the voltage pulses from the two bead populations can be easily distinguished, as is apparent from the histogram in Figure S7 (Supplementary Info). Using this mixture of beads, we carried out the simultaneous detection of serum containing different concentrations of CRP as well as different concentrations of gold nanorods (Nanopartz Inc.) functionalized with streptavidin (SAv). The aggregation ratio can be determined for each of the bead populations separately, by using different analysis windows, namely 0 to $0.05 \mathrm{~V}$ for the smaller anti-CRP beads, and 0.05 to $0.5 \mathrm{~V}$ for the larger biotin beads (Fig. S7). We use the same analysis method outlined above to extract the aggregation ratio for each bead subpopulation. Figure 6a shows the aggregation response of the anti-CRP beads as a function of CRP concentration, for two different SAv concentration levels. In the absence of SAv-functionalized nanorods, the measured aggregation ratio is similar to what was measured previously (Figure 5c). Small variations are expected, as we are now dealing with a mixture of two different bead sizes with different surface modifications. 

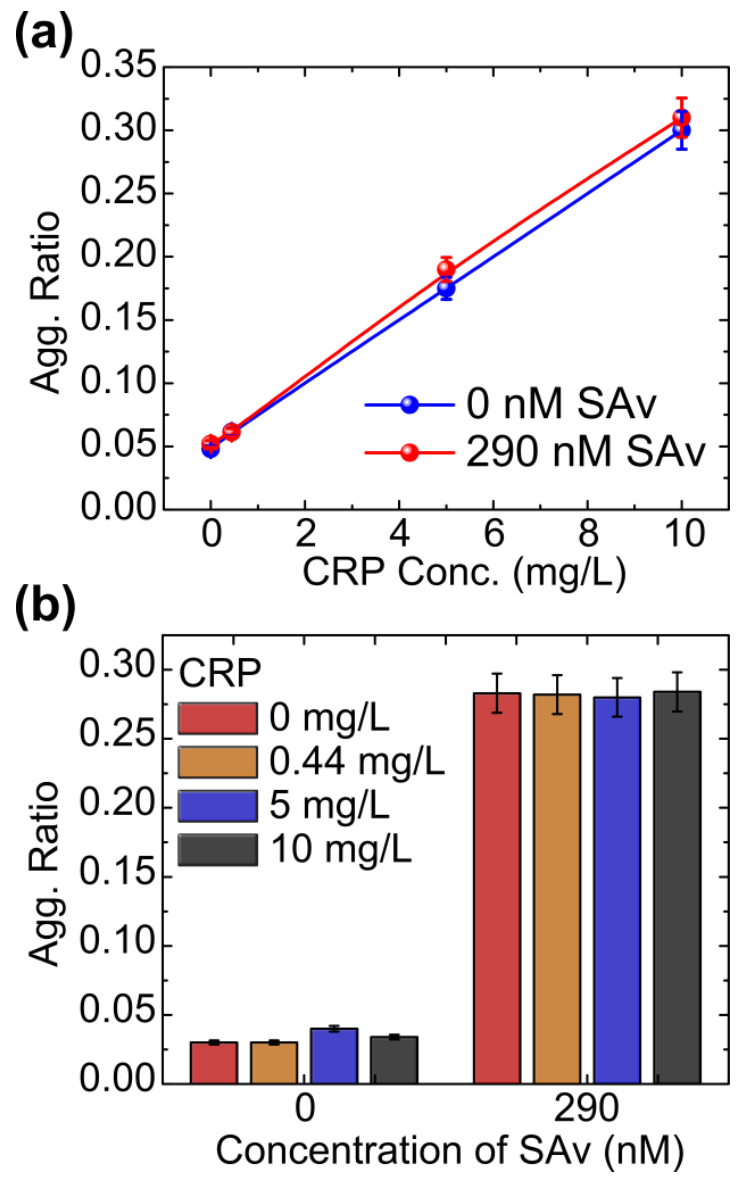

Figure 6

Figure $6 \mathrm{a}$ also shows that the measured aggregation is only slightly affected by the presence of SAv nanorods $([\mathrm{SAv}]=290 \mathrm{nM})$, indicating little interference from SAv in the binding to and aggregation of the anti-CRP beads. The response of the biotin beads is also uncorrelated with the concentration of CRP present in the sample, as is evident from Figure 6b.The response curve for the biotin beads in the presence of SAv is available in the supplementary information. The results in Figure 6 clearly demonstrate that with carefully-chosen receptors, cross-interference can be kept to a minimum, and the aggregation of each bead population can be analyzed separately. In this particular case, we looked at bead sizes with widely spaced monomer peaks, which made the aggregation analysis similar to what we did for the individual bead assays. This method, slightly modified, can be applied to overlapping particle distributions, as long as individual monomer peaks can be distinguished from one another, by monitoring by how much the monomer concentration of each bead population is reduced by aggregation, as a function of analyte 
concentration. The multiplexing capability of the AAC can be increased further by utilizing additional parallel fluidic channels with different constriction sizes, greatly increasing the range of bead sizes that can be analyzed and therefore the number of proteins that can be simultaneously detected.

\section{CONCLUSIONS}

We have demonstrated an on-chip microfluidic device for the label-free, multiplexed detection of proteins in serum, requiring little sample preparation and using a simple measurement setup with an inexpensive, disposable microfluidic analysis chip. We have evaluated the performance of our microfluidic aggregation analyzer with a commercial immunoturbidimetric assay (hsCRP Assay, Diazyme Inc.) and found that our device shows a better LOD and linearity as compared to optical transmittance measurements. Even though the sensitivity of aggregation assays is not as good as ELISA based ones (typically 100x better), there is still a significant market for immunoturbidimetric assays which are simpler and faster than ELISA. Our platform represents a new way of using such commercially available assays without requiring a bulky and expensive optical measurement setup, thereby making immunoturbidimetric measurements even cheaper and potentially suitable for point-of-care (POC) applications. Significantly, the volume of serum required for protein detection is much smaller than that used in turbidimetric measurements, which is always of interest in clinical applications. Furthermore, we demonstrate the potential for multiplexed protein detection by combining two different bead sizes, functionalized with different receptors, and carry out the simultaneous detection of CRP and SAv-coated gold nanorods within the same sample, with minimal cross-interference. This paves the way for a multiplexed lab-on-a-chip aggregation analysis platform, where the instrument presented here could be extended to more than one constriction size/fluidic channel, resulting in a wider range of applicable bead diameters and further multiplexing capabilities. To achieve the goal of a true POC system, on-chip mixing needs to be integrated with the device as well as a filtering stage so that whole blood samples can be used directly. 


\section{ACKNOWLEDGMENTS}

The authors gratefully acknowledge financial support from the W. M. Keck Foundation (SB110056) and DARPA (W911NF-11-1-0228). Optical characterization was made possible by the facilities at the Materials Research Laboratory (MRL) at UC Santa Barbara and the CNSI Biological Nanostructures Lab (UC Santa Barbara). The MRL Shared Experimental Facilities are supported by the MRSEC Program of the NSF under Award No. DMR 1121053, a member of the NSF-funded Materials Research Facilities Network. The authors acknowledge the use of the Biological Nanostructures Laboratory within the California NanoSystems Institute, supported by the University of California, Santa Barbara and the University of California, Office of the President. We are also grateful to the staff of the UC Santa Barbara Nanofabrication Facility for assistance with fabrication and to Joerg Bochmann, Kevin Satzinger, Amit Vainsecher and Greg Peairs, for helpful discussions. 


\section{REFERENCES}

Carbonaro, A., Sohn, L.L., 2005. Lab on a Chip 5, 1155-1160.

Coulter, W.H., 1956. Proc Natl Electron Conf 12, 1034-1040.

Deblois, R.W., Bean, C.P., 1970. Review of Scientific Instruments 41, 909-916.

Deblois, R.W., Wesley, R.K.A., 1977. Journal of Virology 23, 227-233.

Fraikin, J.L., Teesalu, T., McKenney, C.M., Ruoslahti, E., Cleland, A.N., 2011. Nature Nanotechnology 6, 308-313.

Fujita, S., Sano, T., Katayama, Y., 1994. Journal of Clinical Laboratory Analysis 8, 385390.

Hay Burgess, D.C., Wasserman, J., Dahl, C.A., 2006. Nature 444, 1.

Heidelberger, M., Kendall, F.E., 1935. The Journal of Experimental Medicine 61, 563591.

Molina-Bolivar, J.A., Galisteo-Gonzalez, F., 2005. Journal of Macromolecular SciencePolymer Reviews C45, 59-98.

Ozkumur, E., Needham, J.W., Bergstein, D.A., Gonzalez, R., Cabodi, M., Gershoni, J.M., Goldberg, B.B., Unlu, M.S., 2008. Proceedings of the National Academy of Sciences of the United States of America 105, 7988-7992.

Pearson, T.A., Mensah, G.A., Alexander, R.W., Anderson, J.L., Cannon, R.O., Criqui, M., Fadl, Y.Y., Fortmann, S.P., Hong, Y., Myers, G.L., Rifai, N., Smith, S.C., Taubert, K., Tracy, R.P., Vinicor, F., 2003. Circulation 107, 499-511.

Pepys, M.B., Hirschfield, G.M., 2003. Journal of Clinical Investigation 111, 1805-1812.

Phillip, A.G.S., Andrews, P.A., 1987. Journal of Pediatrics 110, 263-265.

Rodriguez-Trujillo, R., Mills, C.A., Samitier, J., Gomila, G., 2007. Microfluidics and Nanofluidics 3, 171-176.

Sabatine, M.S., Morrow, D.A., de Lemos, J.A., Gibson, C.M., Murphy, S.A., Rifai, N., McCabe, C., Antman, E.M., Cannon, C.P., Braunwald, E., 2002. Circulation 105, 17601763 .

Singer, J.M., Vekemans, F.C., Lichtenbelt, J.W., Hesselink, F., Wiersema, P.H., 1973. Journal of Colloid and Interface Science 45, 608-614. 
Sista, R., Hua, Z.S., Thwar, P., Sudarsan, A., Srinivasan, V., Eckhardt, A., Pollack, M., Pamula, V., 2008. Lab on a Chip 8, 2091-2104.

Sridhar, M., Xu, D.Y., Kang, Y.J., Hmelo, A.B., Feldman, L.C., Li, D.Q., Li, D.Y., 2008. Journal of Applied Physics 103, 104701.

Tudos, A.J., Besselink, G.A.J., Schasfoort, R.B.M., 2001. Lab on a Chip 1, 83-95.

Wang, J., 2006. Biosensors \& Bioelectronics 21, 1887-1892.

Wiklund, M., Nord, O., Gothall, R., Chernyshev, A.V., Nygren, P.A., Hertz, H.M., 2005. Analytical Biochemistry 338, 90-101.

Wilson, J.V., Morison, R.A.H., Wright, V., 1960. Journal of Clinical Pathology 13, 453455 .

Wilson, R., 2013. Expert Review of Proteomics 10, 135-149.

Wolf, M., Juncker, D., Michel, B., Hunziker, P., Delamarche, E., 2004. Biosensors \& Bioelectronics 19, 1193-1202.

Yager, P., Domingo, G.J., Gerdes, J., 2008. Annual Review of Biomedical Engineering $10,107-144$.

Zhe, J., Jagtiani, A., Dutta, P., Hu, J., Carletta, J., 2007. Journal of Micromechanics and Microengineering 17, 304-313. 


\section{FIGURE CAPTIONS}

Figure 1 - Principle of the aggregation assay and detection scheme

(a) The Heidelberger-Kendall (H-K) curve (blue) shows how the percentage of aggregates typically varies with antigen concentration.

(b) Measurement scheme of the aggregation analyzer chip (AAC). As particles flow through the constriction, they displace the conducting fluid within the constriction, increasing its associated electrical resistance, which can be correlated to the volume.

Figure 2 - Microfluidic particle analyzer design

(a) Schematic of the microfluidic particle analyzer where the cross-hatched regions indicate in-line filtering areas. Optical micrographs of the fluidic resistor (FR) and micro-constriction (MC) are also shown. The white scale bars for the FR and MC represent 20 microns and 5 microns respectively. (b) Electrical equivalent circuit of the particle analyzer, where the conducting fluid is represented in blue and metal is represented in black. A particle in the MC causes R2 to increase, which causes the voltage at $\mathrm{V}_{\mathrm{s}}$, coupled through the Debye capacitance $\left(\mathrm{C}_{\mathrm{dl}}\right)$ to the solution, to increase.

Figure 3 - Size characterization of polystyrene beads

(a) Voltage pulse due to the passage of a single 0.75 um dia. polystyrene bead through the microconstriction, showing very good signal-to-noise ratio and time resolution. The peak voltage $\Delta \mathrm{V}_{\text {out }}$ for each event is determined from these individual pulses.

(b) Representative time trace for a mixture of polystyrene beads with three different diameters $(0.54,0.75$ and $1.0 \mathrm{um}$ ) measured by the particle analyzer. The histogram on the right is the compilation of pulse heights from the time traces; the three different bead diameters can be clearly identified and from a trimodal Gaussian fit the mean peak voltage signal $\left\langle\Delta \mathrm{V}_{\text {out }}\right\rangle$ for each bead diameter can be determined. 
(c) Mean peak voltage signal $\left\langle\Delta \mathrm{V}_{\text {out }}{ }^{1 / 3}\right.$ for four different bead sizes, plotted as a function of the bead diameter. The excellent linear relationship between $\left\langle\Delta \mathrm{V}_{\text {out }}{ }^{1 / 3}\right.$ and bead diameter confirms that the signal $\Delta \mathrm{V}_{\text {out }}$ is proportional to bead volume, as expected.

Figure 4 - Biotinylated BSA induced aggregation of streptavidin coated beads

(a) Histogram of streptavidin (SAv) beads (0.45 um dia.) in PBST in the absence of biotin-BSA (BBSA), after an incubation at room temperature of 20 minutes. The threshold voltage $\left(\mathrm{V}_{\mathrm{th}}\right)$ for aggregation analysis is set at $0.011 \mathrm{~V}$.

(b) Histogram of SAv beads in PBST after incubation for 20 mins with $1 \mathrm{ug} / \mathrm{mL}$ BBSA, showing an increase in the number of aggregates.

(c) Optical transmittance measurements $(550 \mathrm{~nm})$ for three different concentrations of BBSA, carried out on a UV-Vis spectrophotometer. In the presence of BBSA, the transmittance was found to increase with time, with the initial rate of change of transmittance being proportional to the concentration of BBSA. (d) Comparison of all aggregation ratios measured with AAC to turbidity measurements made with UV spectrophotometer, showing excellent agreement between the two methods. From the SAv bead supplier's datasheet, the binding capacity is estimated to correspond to an equivalence point of $0.005 \mathrm{mg} / \mathrm{mL}$ of BBSA (dashed line). Error bars represent SD from $n=3$ measurements.

Figure 5 - Measurement of C-reactive protein (CRP) in serum by aggregation analysis

(a) Histogram of anti-CRP beads (Diazyme Inc.) in PBST with no added CRP serum; incubation time of 20 minutes. The threshold voltage $\left(\mathrm{V}_{\text {th }}\right)$ is set at $0.01 \mathrm{~V}$ for aggregation analysis.

(b) Histogram of anti-CRP beads in PBST following addition of serum sample containing 10mg/L CRP, followed by incubation for 20 minutes at room temperature. Histogram shows larger number of signals above $\mathrm{V}_{\mathrm{th}}$, indicating the appearance of aggregation induced by CRP. 
(c) Aggregation ratio as determined by the aggregation analyzer chip as a function of CRP concentration. The limit of detection is estimated to be $0.18 \mathrm{mg} / \mathrm{L}$ with good linearity $\left(\mathrm{R}^{2}=0.9986\right)$ to the highest CRP concentrations (Error bars: SD, $\mathrm{n}=3$ ).

(d) Change in optical transmittance at $570 \mathrm{~nm}$ between $\mathrm{t}=5$ minutes and $\mathrm{t}=10$ minutes for the anti-CRP beads, plotted as a function of CRP concentration. The limit of detection (LOD) is $0.88 \mathrm{mg} / \mathrm{L}$ for the measurement carried out at room temperature (Error bars: $\mathrm{SD}, \mathrm{n}=3$ ).

Figure 6-Multiplexed detection of C-reactive protein and streptavidin

(a) Aggregation ratio of the anti-CRP beads as measured by the AAC as a function of CRP concentration at two streptavidin (SAv) levels, 0 and $290 \mathrm{nM}$. The response to the CRP present in the serum sample is minimally affected by the presence of SAv-functionalized nanorods and the response is consistent with our previous measurements of anti-CRP beads only (Error bars: SD, $\mathrm{n}=3$ ).

(b) Bar plot showing the aggregation ratio of the biotin beads at two SAv levels, for different concentrations of CRP in the serum sample. Again, no cross-interference is observed and the response is consistent with the separate bead aggregation measurements (Error bars: SD, $\mathrm{n}=3$ ). 